\title{
Microbiota Research: From History to Advances
}

\author{
Zhengyu $\mathrm{Lu}^{1}$ \\ ${ }^{1}$ College of animal sciences Zhejiang University, China
}

\begin{abstract}
Microbiota are communities of diverse microorganisms found in multicellular creatures. The study of microbiota in human bodies initiated since the late of the 19th century, and has been flourishing with the proceeding of the Human Microbiome Project (HMP), an international project aimed to identify the types and roles of the microorganisms in human. So far, human microbiota, especially the microbiota residing in human intestines, have been shown to affect various aspects of human life, including metabolism, immunity, neural activities, aging process, and so on. Some of them are pathogenic, while some of them are beneficial, or even essential to human. In this review, we summarized the up-to-date studies characterizing the influence of different microorganism to human.
\end{abstract}

\section{Introduction}

Humans are actually "supraorganism"[1]. Instead of selfregulation, human physiological behavior could also be affected by the complex microbiome in the body, which includes bacteria, archaea, fungi, and even protozoans. Bacteria are by far the most numerous members of the human microbiome, which are two or three orders of magnitude more than eukaryotes and archaea, and are one of the most interesting subjects in the human microbiome. In 2016, Israeli and Canadian researchers conducted an accurate estimate of human bacteria and cells, and they then concluded that the number of bacteria of a "reference man" weighing $70 \mathrm{~kg}$ and $1.7 \mathrm{~m}$ heights is about $3.8 \cdot 10^{13}$ and the total mass is $0.2 \mathrm{~kg}$, while the number of human cells is about $3.0 \cdot 10^{13}$. So, the ratio of bacteria to human cells is about 1.3:1[2]. To be noted, the human cells here include red blood cells. If we just count nucleated cells, the ratio is as high as 10:1[2].

Microbes are found all over the body, including the interior and exterior surfaces of gastrointestinal tract, skin, saliva, oral mucosa and conjunctiva [2]. Among them, the gut is the site of the highest diversity and richness of the microbiome. Due to the rich flora, the intestinal tract became an important place for human digestion and absorption: the microbiome that lives in the gut enables the body to have metabolic functions that it does not have. For example, Lactobacillus caseolata reduces aflatoxins, gibberellin, and nitrite in the body. Among them, Aflatoxins cause severe DNA mutagenesis and are highly toxic to liver cancer. Corn gibberellin could make estrogen imbalance, on the female reproductive system. Nitrite in the intestinal acid environment is easy to convert into nitrosamines, these nitrosamines are carcinogenic factors. Other intestinal microorganisms could participate in the metabolism of oral drugs, Zimmermann et al. investigated the ability of 76 human intestinal

$$
\text { Escherichia coli were found Mei's hypothesis: yogurt "the neglected }
$$
in intestines brings a long life

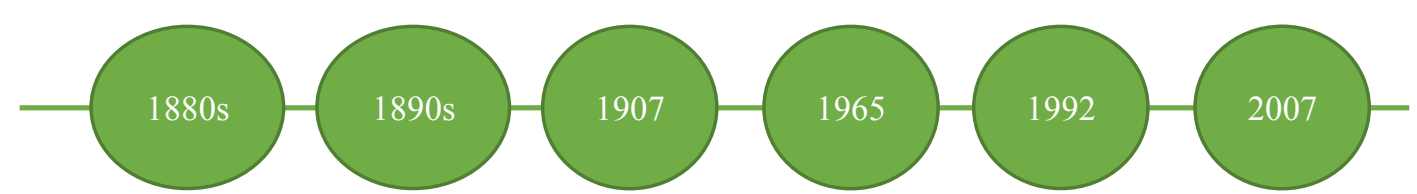

Veillonella and bifidobacteria were isolated
Microscopic images of intestinal microorganism were obtained

\begin{abstract}
Human Microbiome Project (HMP) was launched
\end{abstract}

Figure 1. History of microbiota study

bacteria to metabolize 271 oral drugs. They found that about two-thirds (176 drugs) were metabolized by at least one strain [3]. On the other hand, the disorder of intestinal flora is closely related to the occurrence of various diseases. In addition to being closely related to the digestive system and metabolism [4-7], intestinal flora

* Corresponding author: 3170100524@zju.edu.cn 
could also affect nerve function [8-12], aging [13-15], and even the immune system [16-21]. According to the summer meeting of the nutrition society held in University of Glasgow in 2000, intestine is one of the largest immune organ [22].

Although more and more studies have revealed the importance of intestinal microflora, its diversity and individual differences make the study of intestinal microflora full of challenges. There are about one thousand kinds of intestinal microorganisms, including Firmicutes, Bacteroidetes, Proteobacteria, Actinobacteria, Verrucomicrobia, and Fusobacteria. Among the human intestinal microorganisms, bacteroidetes and firmicutes are the main dominant bacteria[23]. On the other hand, there are great differences in the relative contents of different microbial groups and strains among different host individuals. The factors that affect the difference in microflora include the host's geographic differences, age, physiological status, eating habits, and other factors. In terms of age, according to Claesson's experimental results, the intestinal flora composition of infants and adults is quite different, and with the increase of age, the number of bacteroidetes in the elderly significantly increases [24]. It is worth mentioning that dietary factors are also one of the important factors to change intestinal microorganisms and are the easiest to change or control. Due to different dietary habits, the intake of dietary factors varies greatly among different groups, resulting in great differences in the composition, structure, and function of intestinal microorganisms.

This paper summarizes the important role of intestinal microorganisms in digestion and metabolism, immune circulation, nervous system, and aging. These studies covered different types of microorganisms in different populations to provide references for subsequent microbiome studies.

\section{History of microbiota study}

The first scientific evidence that microbes were part of the normal human body was in the mid-1880s(Figure 1). Austrian pediatrician Theodor Escherich has identified a bacterium in the intestines of healthy children and those affected by diarrhea. It was later named Escherichia coli [25]. The discovery of $E$. coli has laid a foundation for the study of intestinal microorganisms. Soon, Veillonella parvula (1898) [26] and bifidobacteria (1899) [27] were also isolated. Later in the 20th century, many other microbes were isolated from the nasal, oral, skin, gastrointestinal and urogenital tracts and described as part of the human microbiome. But the study of human microbes merely stayed at the conceptual stage.

In 1907, Metchnikoff put forward the famous "Mei's hypothesis" that "yogurt brings a long life" and that lactobacillus could inhibit the growth of intestinal corruption bacteria [28]. The theory for the first time revealed the microorganisms residing in the bodies contributed to the health of the human. It was underestimated for a long time until the mid of the 20th century when it came back to the attention of the mainstream medical field.
In 1965, Dubos et al. published microscopic images of frozen tissue in the stomach of rats, showing many rodshaped or bulbous bacteria attached to the gastric mucosa. This was the first time that human beings obtained microscopic images of intestinal microorganisms in the mucosa of the gastrointestinal tract [29]. Dubos found that in contrast to Lactobacilli and anaerobic Streptococci that were seen in the stomach and both the small and large intestines, the bacteroides group was only found in the large intestine. Moreover, the levels of these three bacterial species were almost constant throughout the life cycle of healthy animals, which suggested their importance and irreplaceability. Other experiments on rats and young pigs have shown that these animals also have high levels of lactic acid bacteria in their gastrointestinal tract. Dubos believed that some components of gastrointestinal flora have achieved symbiosis with the host in the process of evolution, which constitutes the true endophytic flora. Dubos' discovery confirmed the presence of bacteria in living organisms and prompted further research on living bacteria.

In 1992, Bocci first proposed that intestinal microflora had the same metabolic function as virtual organs, and regarded it as "the neglected human organ". Researchers gradually realized the importance of intestinal microflora as a whole to host the intestinal tract. Lipopolysaccharide is a major component of the outer membrane of gramnegative bacteria' cell walls. Bocci pointed out that the traces of lipopolysaccharide secreted from microbiota could activate the immune system and prevent the infection and cancer of the hosts. Furthermore, some evidence suggested that the lipopolysaccharide was the trigger of IFN and other cytokines. Since then, the research community started to value the special function of microbiota and its role in maintaining the healthy state of eukaryotes after this study[30].

An international project, the Human Microbiome Project (HMP)[31], was launched in 2007. The project was aimed to characterize the microorganism and their roles in the human body via the recent whole-genome sequencing technology. The project greatly advanced the research of microbiota, as around 200 microbiota bacterial member species were newly identified in the first three years of the project. Up to date, increasing microbiota species and their roles in human health are being discovered, and the knowledge of microbiota is expanding rapidly.

\section{Different roles of microbiota}

\subsection{Metabolism}

As mentioned in the introduction, microbiota plays an important role in regulating metabolism by affecting digestion and absorption. Numerous recent studies addressed the tight association between microbiota and metabolism regulation.

For example, obesity has been attributed to different types of the gut microbiome as gut microbes result in obesity by altering the energy the hosts absorbed and stored. In 2017, Liu et al. performed a metagenome-wide 
association study and serum metabolomics profiling in a cohort of lean and obese, Chinese youth. The study found that, among obese youth, the abundance of Bacteroides thetaiotaomicron decreased significantly. On the contrary, feeding $B$. thetaiotaomicron to mice reduced their weight gain and obesity caused by diet [4], indicating the role of B. thetaiotaomicron in maintaining health. In 2019,

Petersen et al. found that the expansion of Desulfovibrio, a kind of anaerobic gram-negative bacteria in the gut, and the loss of Clostridia, a gram-positive bacterium in the gut, were key characteristic of obesity and showed linkage to age-associated metabolic syndrome (MetS). Studies have shown that Clostridia and Desulfovibrio are antogonistic, and if the human immune system improperly used Clostridia as a target through the immunoglobulin IgA antibody produced by $\mathrm{T}$ cells, Desulfovibrio would excessively grow, and thus enhancing the host's absorption of lipid and result in obesity. This also implied that immune control of the microbiome helped maintain beneficial microbial populations and thus inhibit lipid metabolism, which might help treat obesity and metabolic diseases in the future [5].

Gut microbiota also directly affects the conversion between adipocytes. Brown adipose tissue, as well as beige adipocytes, promotes thermogenesis to maintain body temperature when in a cold environment. Recently, $\mathrm{Li}$ et al. found that the decrease of gut microbiota might inhibit the increase of mice uncoupling protein 1 (UCP1) and reduce the white adipose tissue (WAT) browning process. By injecting bacterial metabolite butyrate into their stomachs, the heat production capacity of mice recovered. They concluded that intestinal microorganisms might regulating thermogenesis by regulating fat conversion under cold conditions, and likely be mediated by their metabolites butyrate[6].

Meanwhile, certain types of microorganisms might also contribute to the treatment of metabolic-related disease. For example, a high-salt diet might induce TH17 cells and thus cause autoimmune diseases and induce high blood pressure. Wilck et al. Found that high salt intake affected the intestinal flora of mice, especially the presence of Lactobacillus murinus. The researchers then introduced Lactobacillus spp. into the intestinal tract of mice and found that Lactobacillus spp. could prevent the salt-sensitive hypertension by regulating TH17 cells. They also found in human that moderate salt stimulation led to a drop in gut survival in Lactobacillus spp. and an increase in TH17 cells, which in turn increased blood pressure. Therefore, Lactobacillus spp. in the gut might be a potential tool against salt-sensitive diseases [7].

\subsection{Immunity}

In terms of immunity, microbiota is closely related to the immune system of the organism, which brings both positive and negative effects.

Some microbiota increase the body's immune capacity. For example, Cervantes-Barragan et al. found a probiotic, Lactobacillus reuteri, which could induce $\mathrm{CD}^{+} \mathrm{CD} 8$ alpha alpha ${ }^{+}$double-positive intraepithelial lymphocytes (DP IELs). DP IELs are gut $\mathrm{T}$ cells that participate in various immune responses. However, there were no such cells in germ-free mice, suggesting that the differentiation of these $\mathrm{T}$ cells was related to some intestinal microorganisms. Cervantes-Barragan et al. found that $L$. reuteri promoted the differentiation of DP IELs precursors by producing a specific tryptophan indole derivative. The experiment also revealed that the $L$. reuteri and tryptophan-rich diet might promote a better-tolerated, healthier gut environment[16]. Metabolites of another type of gut microbe also increase the body's immune ability. Dodd et al. found a group of microorganisms called Clostridium sporogenes, which used tryptophan, phenylalanine, and tyrosine to produce 12 aromatic amino acid metabolites, and 9 of them are accumulated in host serum. Subsequently, Dodd et al., genetically manipulated the serum levels of these metabolites in sterile mice and found that mice with elevated metabolites had an increase in immunoglobulin $\mathrm{A}$ in their immune systems and a changing in intestinal permeability[17].

Influenza and cancer do great harm to human beings nowadays. Gut microbes also play a role in fighting influenza and cancer. Primary liver tumors and liver metastasis currently represent the leading cause of cancerrelated deaths, human gut commensal bacteria are important regulators of anti-tumor immunity. Ma et al. found that Clostridium scindens used bile acids as messengers to regulate chemokine CXCL16 level on liver sinusoidal endothelial cells (LSEC), and further controlled the accumulation of $\mathrm{CXCR}^{+} \mathrm{NKT}$ cells and tumor expression in the liver. These findings not only show a link between gut microbes, metabolites, and immune responses but also showed important implications for future cancer treatment research[18]. As of the flu virus, Bradley and Finsterbusch et al. found that in lung stromal cells, interferon (IFN) signals are driven by commensal microbiota. Moreover, IFN signal enhancement could prevent the early replication of the influenza virus in the pulmonary epithelium. Antibiotics could reduce IFN signaling and promote early viral replication, possibly because that they kill beneficial bacteria as well. The study highlighted the interaction between bacteria and viruses and the positive role of microbes in the body's immune system, as well as the importance of rational use of antibiotics[19].

However, the negative effects of intestinal microorganisms also cause serious adverse effects on health. Recent studies have shown that the metabolites of some intestinal microorganisms activate drug toxicity and aggravate adverse drug reactions in patients. Irinote could is a first-line drug for the treatment of metastatic colorectal cancer, but the reactivation of irinote could could lead to adverse drug reactions such as severe diarrhea. Guthrie et al. found that microbiome in the human body reactivated irinote could through an enzyme called betaglucuronidases, which increased the risk of adverse reactions of irinotecan-dependent drugs. Inhibition of these enzymes might alleviate adverse reactions in patients treated with irinotecan-dependent drugs. This study provided a new solution to the problem of adverse drug reactions in patients [20].

In severe cases, gut microbes lead to death. For example, some gut microbes cause serious blood 
infections. Enterococci is a common bacterium in the gastrointestinal tract of land animals. Van Tyne et al. analyzed the genomes of enterococcal strains that have caused bloodstream infection in a hospital in the mid1980 s, and then found that under the action of the host's innate immune system and antibiotics, enterococci produced independent mutations at several sites. These fecal enterococci develop antibiotic resistance, produce toxins and are resistant to acidic environment. The mutant enterococci are resistant to phagocytes, and thus could survive in the blood. They would also evolve in the body, and eventually lead to deadly blood infections. The study also revealed the adverse effects of antibiotic use[21].

\subsection{Neural system}

Gut microbes also affect the development and function of the nervous system through metabolites and other means.

For example, microglia are an important part of the brain's nervous system that help brain development, homeostasis, and pathologies. After treating sterile mice with antibiotics, Thion et al. found that microbiome deficiency had gender-specific and time-specific effects on microglia, and microbiome depletion had acute and long-term effects on microglia characteristics[8].

In terms of function, Schretter et al. found that flies in the sterile state and the ones treated with antibiotics showed hyperkinetic motor behavior, with increased flight speed, daily activities, and shorter pauses. However, having mono-colonization with specific bacteria, including the fly commensal Lactobacillus brevis could relieve the symptoms. The experiment revealed that the intestinal microbe regulated the movement pattern of drosophila by acting on the nervous system, and suggested that gut flora might be important for basic behavior in animals[9]. Besides fruit flies, studies have shown that gut microbes enhance exercise time in humans. Scheiman et al. discovered that Veillonella is more prevalent among runners than non-runners through data analysis. After observing the marathon runners, it was found that the relative abundance of Veillonella increased after the marathon. Scheiman et al. extracted a strain of Veillonella atypica from a stool sample, and inoculated it into mice. They then found that the inoculated mice's exhaustive treadmill run time increased significantly. Studies have shown that Veillonella atypica uses lactic acid as the only carbon source, and increase the time of exercise through using its metabolic conversion of exercise-induced lactate into propionate[10].

Gut microbes have also been linked to a variety of neurological diseases. Eran Elinav et al. found that sterile mice and mice treated with antibiotics developed some symptoms of Amyotrophic lateral sclerosis(ALS) such as pre-symptomatic, vivarium-dependent dysbiosis and altered metabolite configuration. After adding 11 species of symbiotic bacteria respectively to the mice treated with antibiotics, Eran Elinav et al. found that Akkermansia muciniphila in the gut relieved ALS symptoms, while the addition of Ruminococcus torques and Parabacteroides distasonis exacerbated ALS symptoms. This study revealed the relationship between ALS and gut microbes and that between the microbial community and the brain[11]. Besides, intestinal microbes might be involved in the production of anxiety, and anxiety symptoms could be transferred with the intestinal flora. Irritable bowel syndrome (IBS) is the most common gastrointestinal disease and is frequently accompanied by comorbid anxiety. De Palma et al. extracted fecal flora from mice with IBS with diarrhea(IBS-D), and transplanted them into sterile mice. Comparing the sterile mice that received IBS-D fecal flora with the control sterile mice, they found that IBS-D mice showed different serum metabolomic profiles, while showing faster gastrointestinal transit, intestinal barrier dysfunction, innate immune activation, and anxiety-like behavior, demonstrating that intestinal flora might affect IBS and accompanying anxiety. Based on this study, it might be possible in the future to treat IBS patients with targeted intestinal flora to relieve diarrhea and anxiety symptoms[12].

\subsection{Others}

Gut microbes also have other different effects on the host.

Gut microbes play a role in the aging process. Bing Han et al. screened 3,983 E. coli mutants and found that the deletion of 29 genes extended the life of host Caenorhabditis. They also noticed that five bacterial mutants promoted longevity of host through increased secretion of the polysaccharide colanic acid (CA). This study revealed molecular targets for developing prolongevity microbes and a bacterial metabolite acting on host mitochondria to promote longevity. It laid a foundation for revealing the influence mechanism of intestinal microorganisms on the human lifespan in the future[13].

Aging in humans is linked to insulin resistance. Bodogai et al. found insulin resistance, which is linked to aging in older mice, is regulated by the changes in gut microbiota[14]. Bodogai et al. thought that reduction in intestinal butyrate and subsequent loss of Akkermansia muciniphila led to changes in intestinal permeability, which led to increased loss of proinflammatory factors that activated monocytes and further impaired insulin signaling pathways. Experiments have also shown that this pathological process is reversible. In the future, it might be possible to reverse this process in humans and delay aging. Meanwhile, another study showed that Akkermansia muciniphila might also be related to premature aging. In two mouse models of premature aging, Barcena et al. found intestinal flora dysregulation, as there was an increase in the abundance of Proteobacteria and Cyanobacteria, and a decrease in the abundance of Verrucomicrobia. Barcena et al. also found that after fecal transplants, premature aging mice that had been transplanted with feces from wild mice lived longer. They noticed that transplantation with the verrucomicrobia (a newly classified type of bacteria commonly found in feces) and Akkermansia muciniphila was sufficient to exert beneficial effects. This experiment indicated that aging is related to intestinal microorganisms, and human intervention could reduce negative effects, providing direct evidence for microbiological intervention in 
aging[15].

Pathological alopecia has always been a common disease. Interestingly, a recent study showed that pathological alopecia might also and the imbalance of intestinal flora. Atsushi Hayashi et al. found that mice treated with Vancomycin could cause intestinal flora disorders, especially the overgrowth of Lactobacillus murinus, this kind of bacteria cannot produce biotin, which depletes available biotin in the gut, eventually leads to pathological alopecia. Supplementation of biotin could reverse hair loss symptoms, suggesting that L. murinus induced hair loss through a biotin-dependent manner. The study laid the groundwork for an unknown link between gut flora and skin diseases[32].

\section{Conclusion}

Until now, a large number and wide variety of microbiota have been found to be closely related to almost all organs of the human body. They play an important role in metabolism, organ function maintenance, disease production, treatment and prevention, and even some trace components of microbial metabolites may affect the normal operation of the whole body. For example, this article introduced the function of microbiota on metabolism, immunity, neural system and etc. With further studies on microbiota species and their functions, we would expect that more connections between microbiota and human bodies to be discovered, which would help us learn human, or the human-microbe supraorganism as a whole better, thus to allow humans living a longer and healthier life. Besides, the research on microorganisms help people learn more about the rational use of antibiotics, which promotes the development of modern biopharmaceuticals and precision medicine.

\section{Reference}

1. Kramer, Peter; Bressan, Paola (2015). "Humans as superorganisms: How microbes, viruses, imprinted genes, and other selfish entities shape our behavior". Perspectives on Psychological Science. 10 (4): 464481. DOI:10.1177/1745691615583131

2. Sender, Ron, Shai Fuchs, and Ron Milo. "Revised estimates for the number of human and bacteria cells in the body." PLoS biology 14.8 (2016): e1002533. DOI: 10.1371/journal.pbio.1002533

3. Zimmermann, Michael, et al. "Mapping human microbiome drug metabolism by gut bacteria and their genes." Nature 570.7762 (2019): 462.

4. "Gut microbiome and serum metabolome alterations in obesity and after weight-loss intervention." Nature Medicine(2017).DOI:10.1038/nm.4358

5. Petersen el al., "T cell-mediated regulation of the microbiota protects against obesity, " Science (2019). DOI: $10.1126 /$ science.aat9351

6. Baoguo Li, Li Li, Min Li, et al. Microbiota Depletion Impairs Thermogenesis of Brown Adipose Tissue and Browning of White Adipose Tissue, Cell Reports (2019). DOI:10.1016/j.celrep.2019.02.015
7. Nicola Wilck, Mariana G. Matus, Sean M. Kearney et al. Salt-responsive gut commensal modulates TH17 axis and disease. Nature, Published online: 15 November 2017, DOI:10.1038/nature24628

8. Morgane Sonia Thion, Donovan Low, Aymeric Silvin et al. Microbiome Influences Prenatal and Adult Microglia in a Sex-Specific Manner. Cell, 25 January 2018, 172(3):500-516, DOI:10.1016/j.cell.2017.11.042

9. Schretter, Catherine E., et al. "A gut microbial factor modulates locomotor behaviour in Drosophila." Nature 563.7731 (2018): 402.

10. Scheiman, Jonathan, et al. "Meta-omics analysis of elite athletes identifies a performance-enhancing microbe that functions via lactate metabolism." Nature Medicine (2019): 1.

11. Eran Elinav et al. Potential roles of gut microbiome \& metabolites in modulation of murine ALS, Nature (2019). DOI: 10.1038/s41586-019-1443-5

12. De Palma, Giada, et al. "Transplantation of fecal microbiota from patients with irritable bowel syndrome alters gut function and behavior in recipient mice." Science Translational Medicine9.379(2017): eaaf6397.DOI:

10.1126/scitranslmed.aaf6397

13. Bing Han, Priya Sivaramakrishnan, Chih-Chun J. Lin et al. Microbial Genetic Composition Tunes Host Longevity. Cell, 15 June 2017, 169(7):1249-1262, doi:10.1016/j.cell.2017.05.036

14. Bodogai, Monica, et al. "Commensal bacteria contribute to insulin resistance in aging by activating innate B1a cells." Science translational medicine 10.467 (2018): eaat4271.

15. Barcena, Clea, et al. "Healthspan and lifespan extension by fecal microbiota transplantation into progeroid mice." Nature medicine 25.8 (2019): 12341242.

16. Luisa Cervantes-Barragan, Jiani N. Chai, Ma. Diarey Tianero et al. Lactobacillus reuteri induces gut intraepithelial CD4 (+) CD8 $\alpha \alpha(+)$ T cells. Science, Published online:03 Aug 2017, DOI:10.1126/science.aah5825

17. Dodd, Dylan, et al. "A gut bacterial pathway metabolizes aromatic amino acids into nine circulating metabolites." Nature (2017).DOI:10.1038/nature246 6

18. Ma, Chi, et al. "Gut microbiome-mediated bile acid metabolism regulates liver cancer via NKT cells." Science 360.6391(2018): eaan5931.

19. Bradley and Finsterbusch et al.: "Microbiota-driven tonic interferon signals in lung stromal cells protect from influenza virus infection" Cell Reports DOI: 10.1016/j.celrep.2019.05.105

20. Guthrie, Leah, et al. "Human microbiome signatures of differential colorectal cancer drug metabolism." Npj Biofilms \& Microbiomes 3.1(2017):27.DOI: $\quad$ 10.1038/s41522017-0034-1

21. Daria Van Tyne, Abigail L. Manson, Mark M. Huycke, et al. Impact of antibiotic treatment and host innate 
immune pressure on enterococcal adaptation in the human bloodstream, Science Translational Medicine (2019). DOI: 10.1126/scitranslmed.aat 8418

22. Arumugam, Manimozhiyan, et al. "Enterotypes of the human gut microbiome." nature 473.7346 (2011): 174.

23. Kelly, D., and A. G. P. Coutts. "Early nutrition and the development of immune function in the neonate." Proceedings of the Nutrition Society 59.02(2000):177-185. DOI: $0.1017 / \mathrm{s} 0029665100000197$

24. Claesson, Marcus J., et al. "Comparative Analysis of Pyrosequencing and a Phylogenetic Microarray for Exploring Microbial Community Structures in the Human Distal Intestine." PLoS ONE 4.8(2009):e6669.dol:10.1371/journal.pone.0006669

25. Shulman, Stanford T., Herbert C. Friedmann, and Ronald H. Sims. "Theodor Escherich: the first pediatric infectious diseases physician?." Clinical infectious diseases 45.8 (2007): 1025-1029. DOI: $10.1086 / 521946$
26. Veillon, André, and A. Zuber. "Recherches sur quelques microbes strictement anaérobies et leur rôle en pathologie." Arch Med Exp 10 (1898): 517-545.

27. Tissier, H. "Le bacterium coli et la reaction chromophile d'escherich." Crit. Rev. Soc. Biol 51 (1899): 943-945.

28. Metchnikoff, Ilya Ilyich. The prolongation of life: optimistic studies. Springer Publishing Company, 2004.

29. Dubos, René, et al. "Indigenous, normal, and autochthonous flora of the gastrointestinal tract." Journal of Experimental Medicine 122.1 (1965): 67-76. DOI: 10.1084/jem.122.1.67

30. Bocci, Velio. "The neglected organ: bacterial flora has a crucial immunostimulatory role." Perspectives in Biology and Medicine 35.2 (1992): 251-260. DOI: 10.1353/pbm.1992.0004

31. http://www.human-microbiome.org/

32. Atsushi Hayashi et al. Intestinal Dysbiosis and Biotin Deprivation Induce Alopecia through Overgrowth of Lactobacillus murinus in Mice, Cell Reports (2017). DOI: 10.1016/j.celrep.2017.07.057 